\title{
A Preliminary Investigation to Decipher an Enigma Using Time-of-Flight Sensor
}

\author{
Rohit Uppal ${ }^{1, a)}$ \\ ${ }^{1}$ Department of Electrical and Computer Engineering, University of Louisville, Louisville, KY
}

a) Corresponding author: ruppalul@gmail.com

Article History

Received : November 2021, Revised : November 2021, Accepted : November 2021, Online : December 2021

\begin{abstract}
Refractive index (RI) was characterized from the angle formed at the axis and slope of the linear fit of the measured perimeter of the loop of a waveguide vs. computed perimeter of the loop of the waveguide by using time-of-flight (TOF) sensor. The RI of uncladded commercially available waveguide was found to be 1.247 and 1.319 at $940 \mathrm{~nm}$ using ToF sensor and ellipsometer, respectively. The novel, simple and cost-effective technique may hold potential to initiate new avenues of research.
\end{abstract}

\section{INTRODUCTION}

A change in refractive index (RI) reflects structural heterogeneity. RI is determined from relative permittivity $(\varepsilon r)$, the absorption coefficient $(\alpha)$ and wavelength $(\lambda)$, as given in equation 1.

$$
n^{2}=\varepsilon_{r}+(\lambda \alpha / 4 \pi)^{2}
$$

For measuring RI, thickness of the films has to be less than a few micrometers and need plane- parallel boundaries while using ellipsometer.[1] For characterizing RI using a confocal optical microscopy, both the phase and group refractive indices would have to be known.[2-5] The measurement depends upon the resolution in a heterodyne interference confocal microscopy.[6,7] Specimen thickness has to be greater than the distance between the foci in case of bifocal optical coherence tomography.[8] Laser meter has been used to ascertain RI.[9]

Time-of-flight (TOF) LiDAR has a laser emitter and single photon avalanche diode (SPAD). SPAD is an avalanche photon diode, which is biased higher than the breakdown voltage. As single photon may trigger an avalanche, hence, SPAD can detect single photon.[10] The distance can be computed by measuring the time accurately between emission and detection of a precisely-controlled pulse.[11]

A novel and simple technique to characterize RI is proposed from the angle formed at the axis and slope of the linear fit of the measured perimeter of the loop of a waveguide vs. computed perimeter of the loop of uncladded commercially available waveguide by using time-of-flight sensor.

\section{MATERIAL AND METHODS}

\section{Commercial Waveguide}

MH Build Series is a clear thermoplastic elastomeric waveguide. It was purchased from MatterHackers, CA. The diameter and the density of the waveguide are $1.75 \mathrm{~mm}$ and $1.12 \mathrm{~g} / \mathrm{cc}$, respectively. 


\section{Wave-Guide Parameter (V)}

A film of the waveguides was fabricated in an oven at $2300 \mathrm{C}$ as discussed elsewhere.[12] The RI of the film was measured by using Woollam ellipsometer (Model HS-190).[12]

\section{Loop Perimeter Computation}

A loop of the uncladded waveguide was connected to time-of-flight sensor (VL53L0X) [Figure 1] at a bias voltage of 3.3V.[12] It is manufactured by STMicroelectronics, Geneva. It has a vertical cavity surface-emitting laser emitter, which emits 3.135 fs pulses with a wavelength of $940 \mathrm{~nm}$ and, and a SPAD. The photodetector is able to detect single photons with a temporal resolution of a few tens of picoseconds. The waveguides were cut by ten centimeters in steps and the loop perimeter was measured. The loop perimeter was computed by the timeof-flight sensor with an 8-bit microcontroller (Arduino Uno).

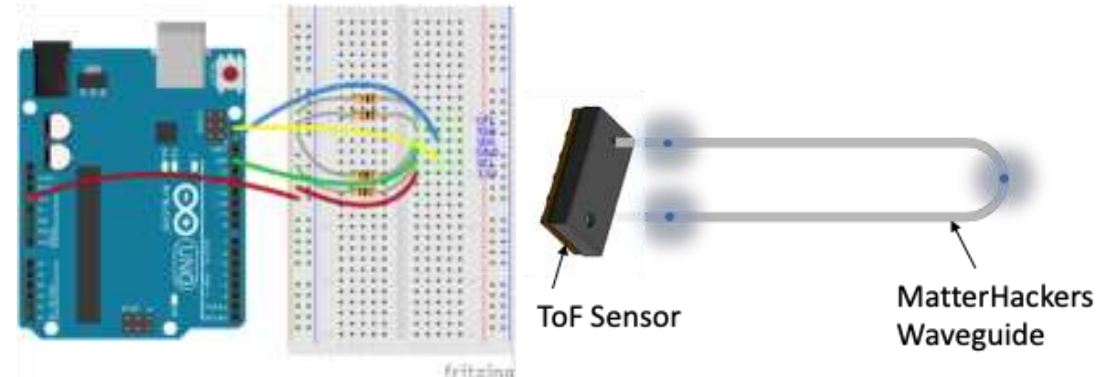

FIGURE 1. Schematic illustration for computation of the perimeter of the loop of a waveguide

\section{RESULTS AND DISCUSSION}

\section{Wave-Guide Parameter}

The RI of the film of waveguides was found to be $1.319 \pm 0.055$ at $940 \mathrm{~nm}$. Highly amorphous film has low number density (N). As RI is square root of relative permittivity or dielectric constant (er). For local field approximation, relative permittivity is a function of polarizability $(\alpha)$ as given in equation (2).

$$
\varepsilon r=\left(1+2 \alpha N / 3 \varepsilon_{0}\right) /\left(1-\alpha N / 3 \varepsilon_{0}\right)
$$

\section{Loop Perimeter and Computation of Refractive Index}

A part of the light is reflected at the interface of the laser emitter and the waveguide, and a part is transmitted. Intensity transmitted through the waveguide is calculated by equation 3 .

$$
I=I_{0} e^{-c \alpha \tau / n L_{m}}
$$

where $\alpha$ is the attenuation coefficient, $\mathrm{Lm}$ is the measured perimeter of the loop of a waveguide, and $\mathrm{c}$ is the speed of light.

It was assumed that in the absence of absorption the intensity of light does not change. Due to the difference in the refractive indices of the waveguide and air, the transmitted fraction is reflected.

Based on the premise that the slope of the linear fit of measured perimeter of the loop of a waveguide vs. computed perimeter of the loop of a waveguide represents a ratio of the flight times in air and in the waveguide, a very simple and novel technique to characterize RI is proposed, as given in equation 4.

$$
n_{w}=c \tau n_{a} \tan \beta_{v} / L_{m} \tan \beta_{w}
$$

Where, bv is the angle formed at the axis by a linear fit of measured distance vs. computed distance in air, bw is the angle formed at the axis by a linear fit of measured perimeter of the loop of a waveguide vs. computed perimeter of the loop of a waveguide (Figure 2), $\tau$ is the time taken by a pulse, which is reflected at the interface 
at the critical angle, to transmit through the waveguide, and nw and na are the refractive indices of the waveguide and air.

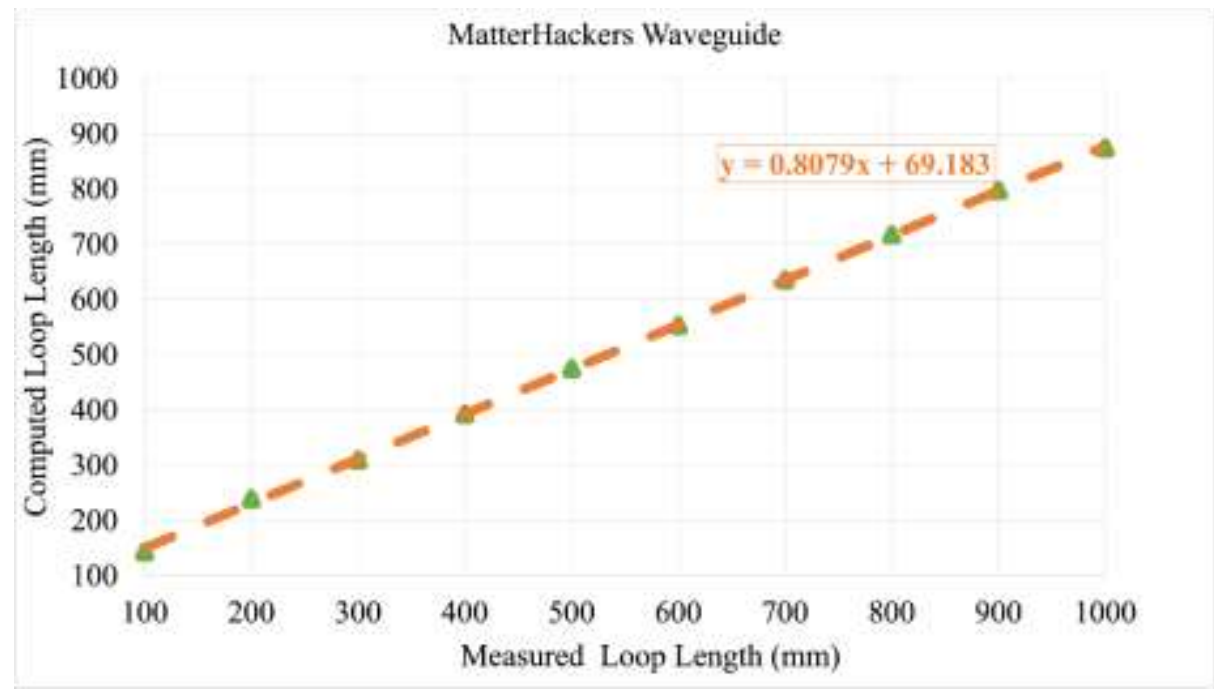

FIGURE 2. Computed distance at different loop perimeters of MatterHackers waveguide.

For air, $\mathrm{Lm}=\mathrm{c} \tau$, and angle formed at the axis by a linear fit of measured distance vs. computed distance in air is $45^{\circ}$. For MatterHackers waveguide, average value of $\mathrm{c} \tau / \mathrm{Lm}$ of the linear fit of the measured perimeter of the loop of the waveguide vs. computed perimeter of the loop of the waveguide is 1.008 (Figure 2 and Table 1). The angle formed by a linear fit of the measured perimeter of the loop of the waveguide vs. computed perimeter of the loop of the waveguide is $38.935^{\circ}$ (Figure 2). The RI of air is 1.000272.[13]

TABLE 1.. Measured and Computed Loop Perimeter of the Waveguide

\begin{tabular}{ccc}
\hline $\begin{array}{c}\text { Measured Loop } \\
\text { Perimeter }(\mathbf{L m})(\mathbf{m m})\end{array}$ & $\begin{array}{c}\text { Computed Loop Perimeter } \\
(\mathbf{L c})(\mathbf{m m})\end{array}$ & $\mathbf{L} \mathbf{c} / \mathbf{L} \mathbf{m}$ \\
\hline 1000 & 874.37 & 0.874 \\
\hline 900 & 797.00 & 0.886 \\
\hline 800 & 717.03 & 0.896 \\
\hline 700 & 635.42 & 0.908 \\
\hline 600 & 552.76 & 0.921 \\
\hline 500 & 474.69 & 0.949 \\
\hline 400 & 392.53 & 0.981 \\
\hline 300 & 309.69 & 1.032 \\
\hline 200 & 238.56 & 1.193 \\
\hline 100 & 143.43 & 1.434 \\
\hline
\end{tabular}

$\mathrm{n}_{\mathrm{w}}=1.008^{*} 1.000272 * \tan \left(45^{\circ}\right) / \tan \left(38.935^{\circ}\right)=1.247$

Hence, the RI of the waveguide (nw) is 1.247. Whereas RI using Woollam ellipsometer was found to be $1.319 \pm 0.055$. The difference in the RI of the waveguide from the proposed method and Woollam ellipsometer could be attributed to that the light pulses within the waveguide propagate in different modes with different group velocities and so, have different flight times. [14] Additionally, it was presumed that the intensity of light does not change in the absence of absorption. 


\section{ACKNOWLEDGEMENT}

I gratefully acknowledge Dr. C.K. Harnett's help for allowing me to use a software. I thank Dr. Martin for measuring the RI and Paul Bupe for drawing a part of the schematic illustration for the computation of the perimeter.

\section{REFERENCES}

1. J.C. Charmet, P.G. de Gennes, J Opt Soc Am 73 (1983) 1777.

2. M.-T. Tsai, F.-Y. Chang, Y.-J. Lee, J.-D. Lee, H.-C. Wang, C.-K. Lee, Opt. Express 19 (2011) 7559.

3. M. Haruna, M. Ohmi, T. Mitsuyama, H. Tajiri, H. Maruyama, M. Hashimoto, Opt. Lett. 23 (1998) 966.

4. M. Ohmi, H. Nishi, Y. Konishi, Y. Yamada, M. Haruna, Meas. Sci. Technol. 15 (2004) 1531.

5. Y. Youk, S. Kim, K. Oh, J. Kobelke, K. Schuster, J. Kirchhof, D.Y. Kim, in:, A. Adibi, S.- Y. Lin, A. Scherer (Eds.), Photonic Crystal Materials and Devices IV, SPIE, 2006, p.61281P.

6. T. Fukano, I. Yamaguchi, Appl. Opt. 38 (1999) 4065.

7. Y. Watanabe, I. Yamaguchi, Appl. Opt. 41 (2002) 4497.

8. S.A. Alexandrov, A.V. Zvyagin, K.K.M.B.D. Silva, D.D. Sampson, Opt. Lett. 28 (2003) 117.

9. K. Aoki, T. Mitsui, Am J Phys 76 (2008) 812.

10. G. Chen, C. Wiede, R. Kokozinski, IEEE Sens. J. 21 (2021) 5656.

11. S. Bellisai, D. Bronzi, F.A. Villa, S. Tisa, A. Tosi, F. Zappa, Opt. Express 21 (2013) 5086.

12. R. Uppal, K. Ajjarapu, K. Kate, C.K. Harnett, Mater Lett 299 (2021) 130079.

13. J.S. Jr, J.H. Zimmerman, (2001).

14. L. Ibbotson, (1998). 